\title{
Improved visualization of virtual-assisted lung mapping by indocyanine green
}

Junko Tokuno, MD, ${ }^{\text {a }}$ Toyofumi F. Chen-Yoshikawa, MD, PhD, a,b Daisuke Nakajima, MD, PhD, ${ }^{\text {a }}$ Akihiro Aoyama, MD, PhD, ${ }^{\mathrm{c}}$ Hideki Motoyama, $\mathrm{MD}, \mathrm{PhD},{ }^{\mathrm{d}}$ Masaaki Sato, $\mathrm{MD}, \mathrm{PhD},{ }^{\mathrm{e}}$ and Hiroshi Date, $\mathrm{MD}, \mathrm{PhD}^{\mathrm{a}}$

\section{ABSTRACT}

Objectives: Virtual-assisted lung mapping (VAL-MAP) is a bronchoscopic marking method of dye application on the surface of the lungs before resecting nonpalpable nodules. However, in some cases, it can be difficult to identify the markings of VALMAP on computed tomography and intraoperative thoracoscopy. We developed and assessed the feasibility of indocyanine green VAL-MAP (ICG-VAL-MAP).

Methods: A historical control trial was conducted to investigate the effectiveness of ICG-VAL-MAP for marking visualization compared with that of VAL-MAP. In ICGVAL-MAP, instead of indigo carmine, ICG and computed tomography contrast agents were used for dye marking, and near-infrared fluorescence endoscopy was employed to visualize the ICG markings. The other processes in VAL-MAP were carried out. The marking visibility was assessed in 3 grades of easy, faint, or not identifiable. We compared the visibility of the markings on computed tomography images and during thoracoscopic operations between VAL-MAP (567 markings in 147 cases) and ICG-VAL-MAP (142 markings in 63 cases).

Results: On the preoperative computed tomography images, ICG-VAL-MAP provided significantly better marking visualization than VAL-MAP (easy/faint/not identifiable $=142 / 0 / 0$ vs 427/100/30; $P<.0001$ ). ICG-VAL-MAP provided significantly better intraoperative markings than VAL-MAP (easy/faint/not identifiable $=141 / 0 / 1$, respectively, vs 475/50/42, respectively; $P<.0001$ ). Regarding complications, pneumothorax occurred in $8(5.4 \%)$ cases of VAL-MAP and zero cases $(0 \%)$ of ICG-VAL-MAP $(P=.12)$; fever was observed in $7(5.0 \%)$ cases of VAL-MAP and $2(3.2 \%)$ cases of ICG-VAL-MAP $(P=.72)$.

Conclusions: ICG-VAL-MAP provided significantly better visibility of markings than VAL-MAP. It might be useful in the resection of nonpalpable small lung lesions. (JTCVS Techniques 2021;10:542-9)

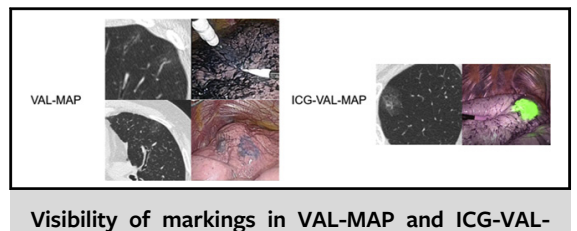
MAP.

\section{CENTRAL MESSAGE \\ ICG-VAL-MAP showed signifi- cantly better marking visualiza- tion on preoperative CT and during thoracoscopic surgery than VAL-MAP.}

\section{PERSPECTIVE}

To improve the visibility of markings of VAL-MAP, we developed ICG-VAL-MAP, employing indocyanine green and $\mathrm{CT}$ contrast instead of indigo carmine as the dye agent. ICG-VAL-MAP provided significantly better marking visualization than VAL-MAP. It may have a positive influence on resection of nonpalpable small lung lesions.

See Commentaries on pages 550 and 552.
From the ${ }^{a}$ Department of Thoracic Surgery, Graduate School of Medicine, Kyoto University, Kyoto, Japan; ${ }^{b}$ Department of Thoracic Surgery, Graduate School of Medicine, Nagoya University, Nagoya, Japan; 'Department of Thoracic Surgery, Kobe City Medical Center General Hospital, Kobe, Japan; ${ }^{\mathrm{d}}$ Department of Thoracic Surgery, Kobe City Nishi-Kobe Medical Center, Kobe, Japan; and ${ }^{\mathrm{e}}$ Department of Thoracic Surgery, Graduate School of Medicine, The University of Tokyo, Tokyo, Japan.

Received for publication Nov 25, 2020; accepted for publication July 30, 2021; available ahead of print Aug 8, 2021.

Address for reprints: Hiroshi Date, MD, PhD, Department of Thoracic Surgery, Graduate School of Medicine, Kyoto University, 54 Shogoin-Kawahara-cho, Sakyo-ku, Kyoto 606-8507, Japan (E-mail: hdate@kuhp.kyoto-u.ac.jp).

2666-2507

Copyright (C) 2021 The Author(s). Published by Elsevier Inc. on behalf of The American Association for Thoracic Surgery. This is an open access article under the CC BY-NC-ND license (http://creativecommons.org/licenses/by-nc-nd/4.0/). https://doi.org/10.1016/j.xjtc.2021.07.019
Video clip is available online.

The incidental finding of small lung nodules has been increasing, owing to the development of imaging technologies. ${ }^{1}$ Now that video-assisted thoracoscopic surgery has become a standard approach, preoperative or intraoperative marking techniques have become necessary for resection of nonpalpable small lesions. Various methods have been developed to help identify the location of such lung nodules. ${ }^{2}$ However, conventional marking techniques, such as computed tomography (CT)-guided percutaneous marking using a hook wire, have possible serious complications, including pneumothorax, hemorrhage, and potentially fatal air embolism. ${ }^{3,4}$ 


\section{Abbreviations and Acronyms \\ CT = computed tomography \\ GGO $\quad=$ ground glass opacity \\ ICG $=$ indocyanine green \\ ICG-VAL-MAP = indocyanine green virtual- assisted ling mapping \\ NIR = near-infrared \\ VAL-MAP $=$ virtual-assisted lung mapping}

Virtual-assisted lung mapping (VAL-MAP) has been established to have a role in navigation during lung surgery, particularly in assisting the localization of nonpalpable small nodules or setting the intersegmental plane in segmentectomy cases. ${ }^{5-9}$ VAL-MAP is a relatively new bronchoscopic multispot dye-marking technique with fewer complications. ${ }^{5-8}$ However, despite its advantages, VALMAP has some limitations, including difficult detection of dye markings in some cases on CT and on the surface of the lung during surgery. ${ }^{6,8}$ Sato and colleagues ${ }^{6}$ reported on the ease of VAL-MAP identification on CT; $17.6 \%$ of the markings were difficult to identify and $5 \%$ were not identifiable. Intraoperatively, $6.0 \%$ of the markings were unidentifiable and $10.2 \%$ were faintly and hardly visible.

In recent years, the most widely used dye in the nearinfrared (NIR) fluorescence technique has been indocyanine green (ICG), which has an excitation of $778 \mathrm{~nm}$ and emission of $830 \mathrm{~nm} .{ }^{10}$ Some reports have indicated the feasibility and benefits of NIR fluorescence image-guided tumor nodule detection during surgery. ${ }^{8,11-15}$ We developed a novel technique of ICG virtual-assisted ling mapping (ICG-VAL-MAP), which is a revised method of VAL-MAP, using ICG and CT contrast enhancing agent instead of indigo carmine as the dye, and hypothesized that ICG-VAL-MAP can improve marking visibility. Moreover, we aimed to assess the efficacy of ICG-VAL-MAP, in comparison with that of the original method.

\section{METHODS \\ Patients}

A historical control trial was conducted at Kyoto University Hospital to validate the efficacy of ICG-VAL-MAP compared with that of VALMAP. Ethical approval for this study was obtained from institutional review board (No. YC1223, January 21, 2019). VAL-MAP data accumulated from July 2012 to December 2014 were used as the historical cohort. Patients who underwent ICG-VAL-MAP followed by lung resection between January 2017 and March 2020 were included in the ICGVAL-MAP group. The exclusion criteria were history of bronchial asthma, renal failure, and allergy to the agents, as well as cases of 2 or more procedures in 1 surgery (eg, multiple wedge resections, segmentectomies, and combined case of wedge resection and segmentectomy). This study was approved by the institutional ethical board of Kyoto University Hospital. Oral and written consent were obtained from all patients.

\section{ICG-VAL-MAP/VAL-MAP Planning and Procedures}

The planning and procedures, except the dye agent, were similar between ICG-VAL-MAP and the original VAL-MAP., (Figure 1). Virtual bronchoscopy (Synapse Vincent; Fujifilm Medical, Tokyo, Japan) was used to identify the target bronchi that would reach the designed marking points and to plan bronchoscopic procedures. Single or multiple markings were set to indicate the lesion for wedge resection cases or the resection lines for both wedge resection and segmentectomy cases.

After planning with virtual bronchoscopy, actual bronchoscopy was conducted under sedation and local anesthesia within 2 days preoperatively. The dye injection technique for VAL-MAP included insertion of a metal blunt-tip catheter (PW-6C-1; Olympus, Tokyo, Japan), which was preloaded with 0.2 to $1.0 \mathrm{~mL}$ indigo carmine per 1 marking, through the working channel of the regular flexible bronchoscope (BF 260; Olympus, Tokyo, Japan) and into the target bronchi, under the guidance of the virtual bronchoscopy images. Thereafter, the dye was gently injected while confirming fluoroscopically that the tip of the catheter reached the visceral pleura and that the dye had spread. ${ }^{5-7}$ The injection procedures were repeated for all target bronchi. For ICG-VAL-MAP, the dye injection technique was the same as that for the original method of VAL-MAP, except that it used $0.1 \mathrm{mg}$ Diagnogreen (Daiichi-Sankyo, Tokyo, Japan) for injection in place of ICG and $66.6 \mathrm{mg}$ Iopamiron (Bayer AG, Leverkusen, Germany) as the CT contrast per 1 marking. The number of markings and selection of the dye agents on each marking depended on the bronchoscopist's or surgeon's preference.

\section{CT Scan after ICG-VAL-MAP/VAL-MAP}

An additional CT scan was performed to confirm the location of markings after the mapping procedure. The ease of identifying each marking on the CT images was divided into 3 grades by the surgeon or bronchoscopist: easy, faint, or not identifiable. For ICG-VAL-MAP, which used a CT contrast agent, the spread markings were contrast-enhanced. The CT images after VAL-MAP were reconstructed into 3-dimensional images, including the target lesion, markings, and other geometric information.

\section{Intraoperative Findings}

All surgical procedures were conducted under thoracoscopy or with the aid of thoracoscopy. In ICG-VAL-MAP, NIR endoscopy was employed to visualize the ICG markings. The ease of identification of each marking was graded as easy, faint, or not identifiable by the surgeon. The type of operation was reported as wedge resection or segmentectomy, including subsegmentectomy or lobectomy.

\section{Data Analysis}

Marking visibility grading was retrospectively conducted using the CT images obtained after ICG-VAL-MAP and intraoperative video recordings. The marking visibility for ICG-VAL-MAP was assessed on the basis of three grades of easy, faint, or not identifiable. The visibility data of the VAL-MAP cohort, which were originally divided into 4 or 6 grades for CT or intraoperative findings, respectively, were rendered into the same 3 grades as those of ICG-VAL-MAP. Clinical information, including lesion size and depth, procedures, and complications, were examined by chart review. All data were collected separately for each case (patient's demographic data and complications), lesion (lesion size and depth, procedure, and pathology), and marking (visibility). Statistical analysis was conducted using GraphPad Prism (GraphPad Software, San Diego, Calif). The data were presented as median (interquartile range [IQR]), and the 2 groups were compared using the $t$ test to examine the differences in tumor size and depth. The $\chi^{2}$ test was used to examine the differences in complications, and the Fisher exact test was used to examine the differences in surgical procedures, radiological features, lesion pathology, and marking visibility. 


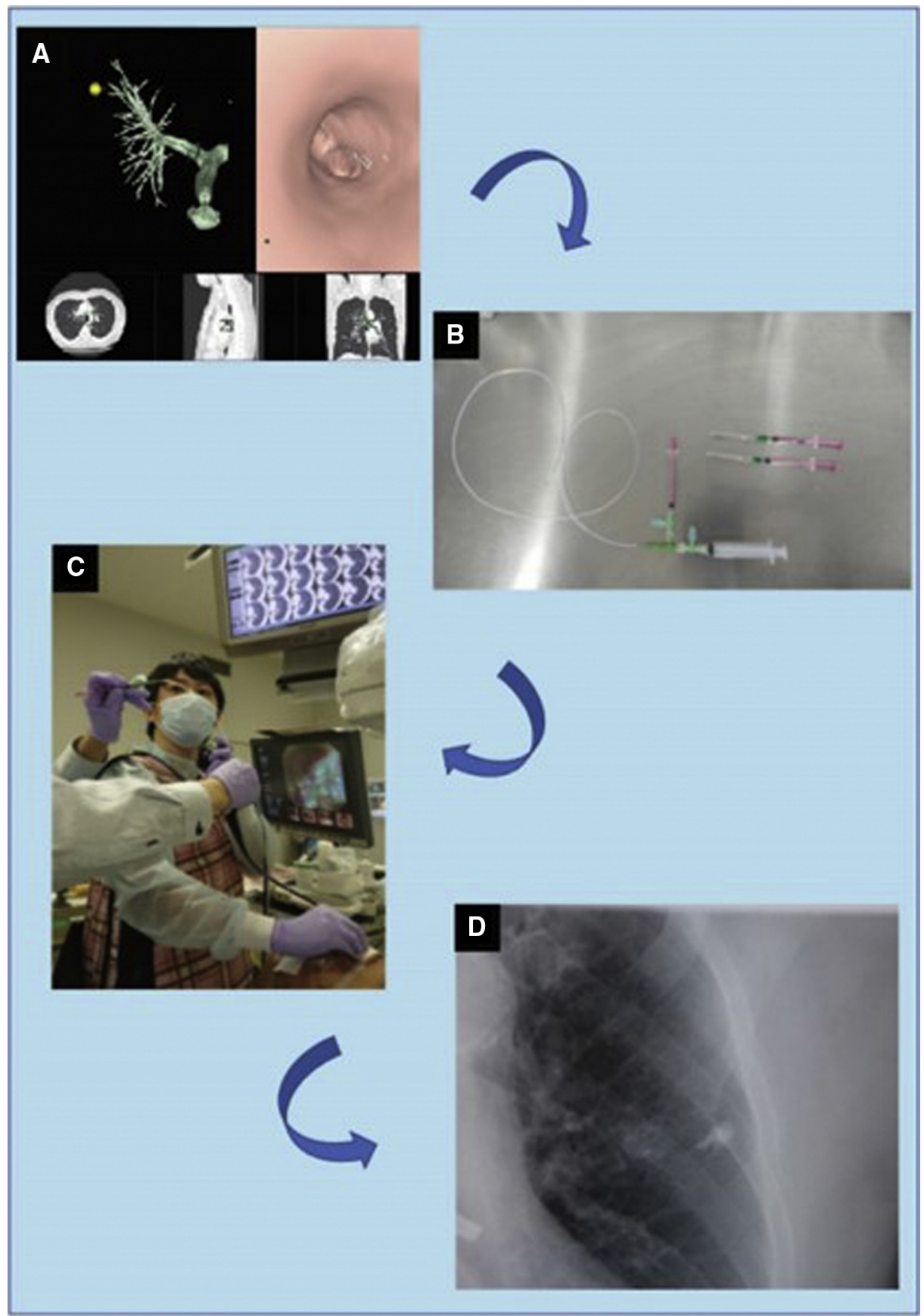

FIGURE 1. Technique flow of indocyanine green virtual-assisted lung mapping (ICG-VAL-MAP). A, Planning on virtual bronchoscopy. B, Catheter and dye agent used for bronchoscopic marking. C, Dye injection through a catheter inserted in the working channel of the bronchoscope. D, Marking procedure performed under fluoroscopic confirmation. Because the dye includes a computed tomography contrast agent, the marking is easily observed fluoroscopically (Video 1).

\section{RESULTS}

Ninety patients were considered eligible for ICG-VALMAP. However, 1 patient was excluded because of operation cancelation, 5 patients were excluded owing to insufficient data on markings, and 21 patients were excluded because a combination of ICG and CT contrast and indigo carmine marking was used in them (Figure 2). In total, 63 patients underwent ICG-VAL-MAP followed by lung resection. The characteristics of the patients, lung lesions, and markings are shown in Table 1 . The ages of the patients who underwent ICG-VAL-MAP were significantly higher than those of the patients who underwent 


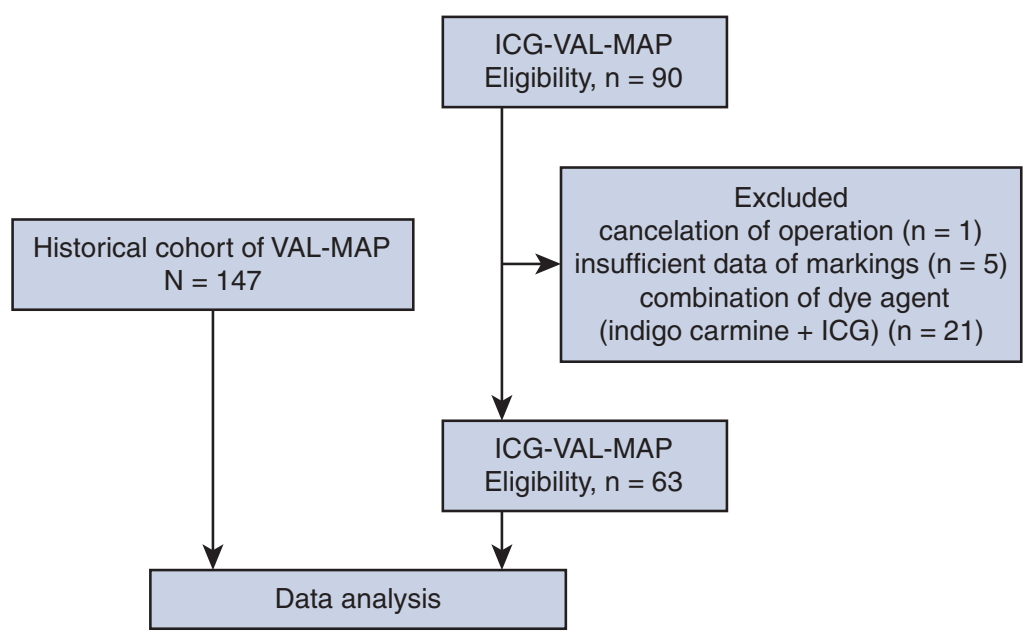

FIGURE 2. Flow diagram of patient participation in the study. As a historical control cohort, we used virtual-assisted lung mapping (VAL-MAP) data collected between 2012 and 2014. For indocyanine green virtual-assisted ling mapping (ICG-VAL-MAP), we collected data from 2017 to 2020 . One patient was excluded because of operation cancelation, 5 were excluded owing to insufficient data on the markings, and 21 were excluded because both dyes, indigo carmine and ICG, were used. ICG, Indocyanine green.

VAL-MAP (65 years vs 69 years; $P=.03$ ). No significant sex-related differences were found between the groups. For the VAL-MAP group $(\mathrm{n}=147), 228$ lesions were targeted and 567 markings were conducted. The median diameter of the lung nodules was $8.0 \mathrm{~mm}$ (IQR, 4.5-12.0 mm) and the median depth from the surface of the lung was $4.0 \mathrm{~mm}$ (IQR, 1.0-10.0 mm). There were 101 wedge resections, 93 segmentectomies, and 3 lobectomies performed. There was 1 nodule that could not be detected and resected. Among the 227 resected nodules with the use of VAL-MAP, the pathologic diagnosis was primary lung cancer in 105 , metastatic lung tumor in 82 , and benign lung nodule in 40. The radiological features were pure ground-glass opacity (GGO), GGO with a solid component, and a solid component alone in 68,35 , and 125 cases, respectively.

For the ICG-VAL-MAP group $(\mathrm{n}=63), 77$ lesions were targeted and 142 markings were conducted. The median diameter of the lung nodules was $8.0 \mathrm{~mm}$ (IQR, 6.0$13.9 \mathrm{~mm}$ ) and the median depth from the surface of the lung was $9.0 \mathrm{~mm}$ (IQR, 2.0-13.0 mm). There were 44 wedge resections, 28 segmentectomies, and 1 lobectomy performed. Regarding the radiological features, 19 lesions

TABLE 1. Characteristics of patients and markings

\begin{tabular}{|c|c|c|c|}
\hline Characteristic & VAL-MAP & ICG-VAL-MAP & $P$ value \\
\hline Age $(y)$ & $65(58.75-72.0)$ & $69(62-75)$ & .03 \\
\hline Sex, male/female & $35 / 28$ & $66 / 81$ & .15 \\
\hline No. of cases & 147 & 63 & N/A \\
\hline No. of targeted lesions & 228 & 77 & N/A \\
\hline Resected nodules & $227(99.6)$ & 77 (100) & $>.99$ \\
\hline Size of lesions $(\mathrm{mm})$ & $8.0(4.5-12)$ & $8.0(6.0-13.9)$ & .82 \\
\hline Depth of lesions (mm) & $4.0(1-10)$ & $9.0(2.0-13)$ & .01 \\
\hline \multicolumn{4}{|l|}{ Radiological feature of the nodule } \\
\hline Pure GGO/GGO with solid component/solid & $68 / 35 / 125$ & $19 / 29 / 29$ & .0003 \\
\hline No. of markings & 567 & 142 & N/A \\
\hline Per nodule & $3(2-4)$ & $2(1-3)$ & $<.0001$ \\
\hline \multicolumn{4}{|l|}{ Surgical procedures } \\
\hline Wedge/segmentectomy/lobectomy & $101 / 93 / 3$ & $44 / 28 / 1$ & .36 \\
\hline \multicolumn{4}{|l|}{ Pathology } \\
\hline Lung cancer/metastasis/benign & $105 / 82 / 40$ & $55 / 18 / 4$ & $<.0001$ \\
\hline
\end{tabular}

Values are presented as $\mathrm{n}, \mathrm{n}(\%)$, or median (interquartile range). VAL-MAP, Virtual-assisted lung mapping; ICG-VAL-MAP, indocyanine green virtual-assisted ling mapping; $N / A$, not available; $G G O$, ground-glass opacity. 
TABLE 2. Visibility of markings of virtual-assisted lung mapping $(V A L-M A P)$ and indocyanine green virtual-assisted ling mapping (ICG-VAL-MAP)

\begin{tabular}{cccc}
\hline Visability & VAL-MAP & ICG-VAL-MAP & $P$ value \\
\hline $\begin{array}{c}\text { In CT images } \\
\text { Easy/faint/not } \\
\text { identifiable }\end{array}$ & $437 / 100 / 30$ & $142 / 0 / 0$ & $<.0001$ \\
$\begin{array}{c}\text { Intraoperative } \\
\text { Easy/faint/not } \\
\text { identifiable }\end{array}$ & $475 / 50 / 42$ & $141 / 0 / 1$ & \\
\hline
\end{tabular}

Values are presented as $\mathrm{n} / \mathrm{n} / \mathrm{n}$. CT, Computed tomography.

were pure GGO, 29 were GGO with a solid component, and 29 were solid lesions. All targeted lesions were successfully resected. Among the 77 resected nodules with the use of ICG-VAL-MAP, the pathologic diagnosis was primary lung cancer in 55, metastatic lung tumor in 18 , and benign lung nodule in 4.

Overall, ICG-VAL-MAP was performed targeting significantly deeper lesions than VAL-MAP $(P=.01)$, and the median number of markings per 1 lung nodule in each case was significantly less in the ICG-VAL-MAP group than in the VAL-MAP group (3, IQR, 2-4 vs 2, IQR, 1-3; $P<.0001)$. The variance of the radiological features and pathology of the lesions were significantly different between the 2 groups $(P=.003$ and $<.0001$, respectively) (Table 1$)$.

\section{Visibility on CT Scan}

In the VAL-MAP group, $437(77.0 \%)$ markings were easily identifiable on CT scan, $100(17.6 \%)$ were identifiable but faint, and $30(5.3 \%)$ were not identifiable. In the ICG-VAL-MAP group, all of the 142 markings were easily identifiable on CT scan. In the analysis of visibility according to 2 grades of visible and not visible, the markings were significantly better with ICG-VAL-MAP than with VALMAP $(P<.0001)$ (Table 2 and Figures 3-6).

\section{Intraoperative Visibility}

In the VAL-MAP group, $475(83.8 \%)$ markings were easily identifiable, $50(8.9 \%)$ were identifiable but faint, and $42(7.4 \%)$ were not identifiable. In the ICG-VALMAP group, $141(99.3 \%)$ markings were easily identifiable and $1(0.7 \%)$ was not identifiable on the lung surface on NIR fluorescence endoscopy. The intraoperative visibility of the markings was significantly better with ICG-VAL-MAP than with VAL-MAP $(P<.0001)$ (Table 2 and Figures 3-6).

\section{Complications}

Pneumothorax and pleural injury were observed in 8 cases in the VAL-MAP group and in no case in the ICG-VALMAP group $(P=.12)$. However, the severity of pneumothorax in each case was mild, and none required chest drainage before the surgery. Fever $\left(>38^{\circ} \mathrm{C}\right)$ during the period from the bronchoscopic procedure to surgery was noted in 7 cases in the VAL-MAP group and in 2 cases in the ICGVAL-MAP group. The other complications were alveolar hemorrhage in 1 patient in the VAL-MAP group and vagal reflex immediately after the procedure in 1 patient in the ICG-VAL-MAP group. In both groups, there was no case that was canceled or postponed because of complications (Table 3). Video 1 describes brief summary of the results.

\section{DISCUSSION}

Surgical simulation and/or navigation have recently been more frequently attempted in the field of thoracic surgery. ${ }^{16,17}$ Among the methods, VAL-MAP plays important roles in the navigation and localization of lung resection ${ }^{5-9}$; however, there remains a room for improvement in visibility on CT images and during operation. Sato and colleagues ${ }^{6}$ cited examples of difficult identification of markings on CT images after VAL-MAP. Because indigo carmine is not radiopaque, markings would be identified as a
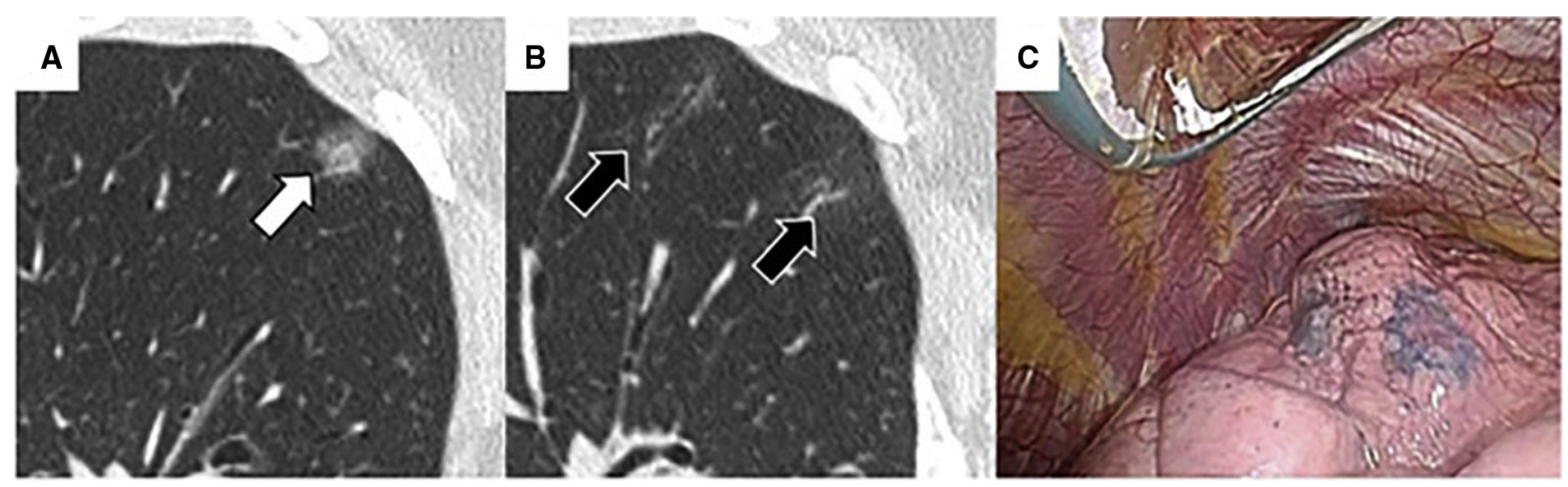

FIGURE 3. A representative case of virtual-assisted lung mapping (VAL-MAP) with easily identifiable markings. A, The target nodule is located in the right upper lobe (white arrow). B, The markings are arranged to surround the nodule and are observed as a consolidation on the computed tomography scan after VAL-MAP (black arrow). C, Intraoperative findings. The blue colored markings with indigo carmine are easily observed on the surface of the lung. 

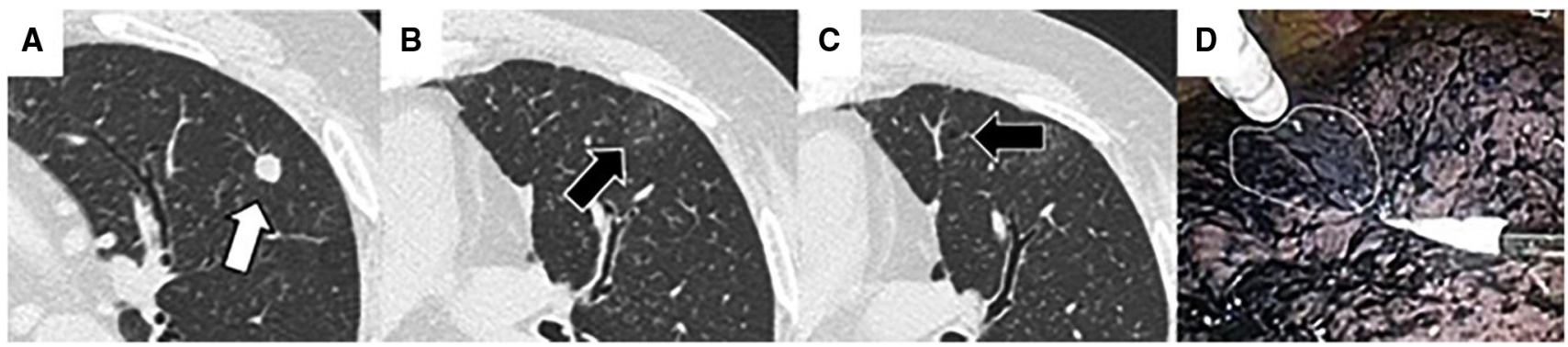

FIGURE 4. A representative case of virtual-assisted lung mapping (VAL-MAP) with faint markings. A, Target nodule located in the left upper lobe (white arrow). B, One marking identified as faint consolidation on computed tomography (CT) scan after VAL-MAP (black arrow). C, Another marking identified as faint bronchiectasis on CT scan after VAL-MAP (black arrow). D, Using VAL-MAP, finding the blue-colored marking with indigo carmine (encircled by the white line) intraoperatively is difficult because of anthracosis on the surface of the lung.

GGO-like appearance on CT. Therefore, faint markings may not be clearly observed in some cases. Moreover, a dorsal location of the GGO-like appearance would make it hard to distinguish between the dye markings and the gravity effect. ${ }^{6}$ Failure to identify the markings would make lung resection planning, such as setting the cutting lines and ensuring the margin, challenging. To overcome this weak point, we intended to add a CT contrast to the dye agent in the current study. Based on the significant improvement in visibility on preoperative $\mathrm{CT}$, this addition of a $\mathrm{CT}$ contrast to the dye might be effective in obtaining accurate information for planning strategies.

Next, we focused on the improvement of intraoperative visibility of the markings on the surface of the lung. Using VAL-MAP, we experienced some difficulties in detecting the markings with indigo carmine, especially in patients with emphysema and interstitial pneumonia, because the color of the lung surface was similar to the color of indigo carmine. Moreover, when the dye injection was performed at a relatively more proximal bronchus, identification of the marking location was difficult. On the other hand, with the use of ICG-VAL-MAP, the ICG markings on intraoperative NIR endoscopy were green in color and provided an obvious contrast against the background tissues. Therefore, visibility was significantly improved with ICG-VAL-MAP.
The number of markings for each nodule was significantly less with ICG-VAL-MAP than with VAL-MAP. This might have been brought about by the improvement of visibility on both CT and intraoperative findings. Having better visibility would need fewer markings and might lead to a shorter time of bronchoscopy. Moreover, despite the relatively fewer number of markings with ICG-VALMAP, all nodules were successfully resected. This may be considered among the advantages of ICG-VAL-MAP.

In this study, we found significant variances in patient age and lesion characteristics such as depth, rate of GGO, and pathology between the groups. However, because each marking itself was performed in the very peripheral bronchi to visualize the relative geometrical information of the nodules on the surface of the lung, the characteristics of the nodules and patients should not affect the visualization of the markings.

Pneumothorax was the main complication of VAL-MAP, whereas none of the patients receiving ICG-VAL-MAP developed complications. Although there was no significant difference between the 2 procedures, we considered 2 reasons for the fewer pneumothorax cases after ICG-VALMAP than after VAL-MAP. First, the CT contrast content of the injected dye used in ICG-VAL-MAP enabled visualization of the spread of the dye to the peripheral bronchi or
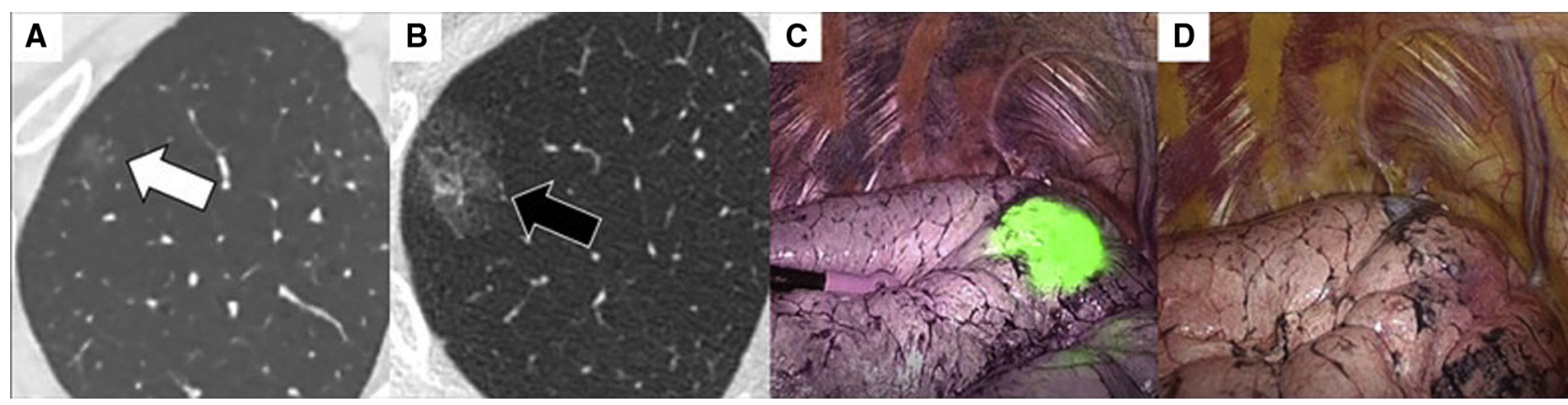

FIGURE 5. A representative case of indocyanine green virtual-assisted ling mapping (ICG-VAL-MAP). A, Target nodule located in the right upper lobe had ground-glass opacity (white arrow). B, Marking with ICG and computed tomography (CT) contrast agent located close to the nodule is observed as an obvious consolidation on CT scan after virtual-assisted lung mapping (VAL-MAP) (black arrow). C and D, Intraoperatively, the marking is easily identifiable in green on near-infrared thoracoscopy (C), but is not identifiable under visible light (D). 


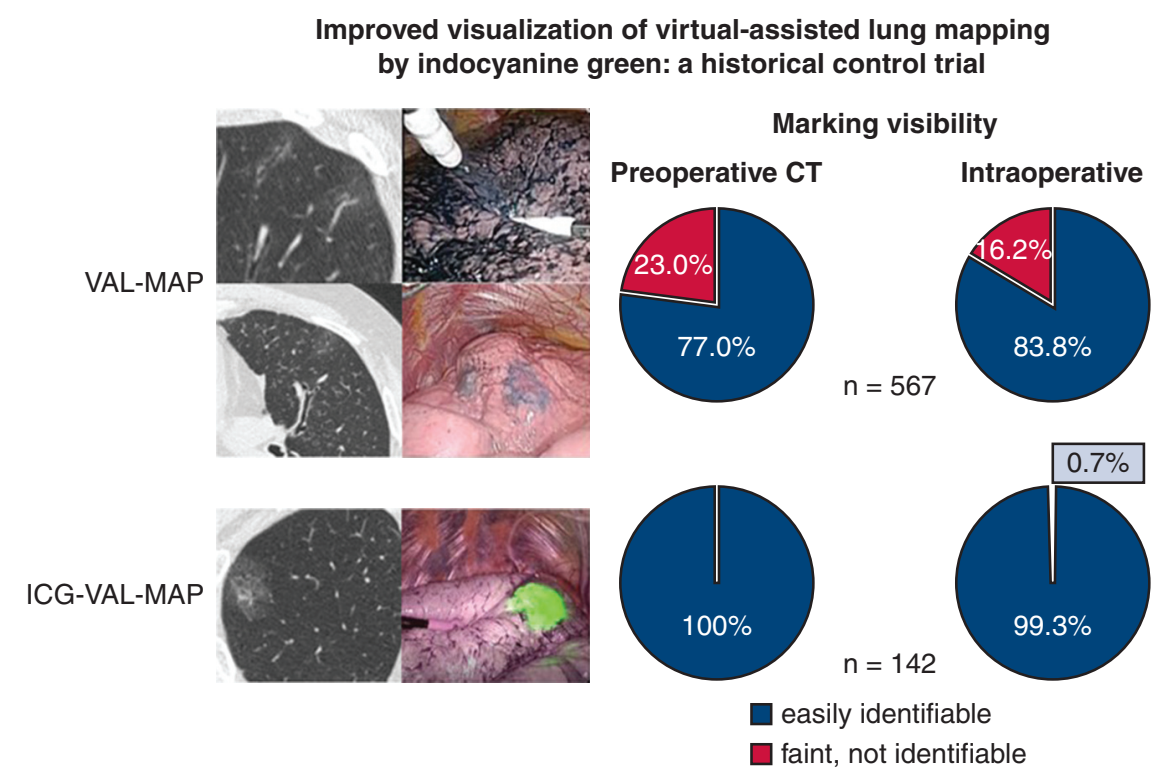

FIGURE 6. Comparison of marking visibility between virtual-assisted lung mapping (VAL-MAP) and indocyanine green virtual-assisted ling mapping (ICG-VAL-MAP). ICG-VAL-MAP showed significantly better marking visualization on preoperative computed tomography $(C T)$ and during thoracoscopic surgery than VAL-MAP.

lung parenchyma under fluoroscopy. Second, because ICG was efficiently visualized intraoperatively, even if it was spread at a certain depth from the surface of the lung, injection to the very peripheral bronchi was not necessary. These features of ICG-VAL-MAP might have helped reduce the risk of pneumothorax.

By far, several marking methods have been invented to help in the resection of small lung nodules. VAL-MAP is among the established techniques that use dye because it does not entail monitoring of the collection or dislocation of the markings. ICG-VAL-MAP would be easy to introduce because the procedure is the same as that of VALMAP, except for the different dye for injection through the bronchi.

The present study had some limitations. First, ICGVAL-MAP is available only when the institution where the procedure takes place has an NIR scope installed. However, because the use of fluorescence-guided operation has become increasingly common in every specialty of surgery, this issue would be solved overtime. Second, the present study was neither a randomized nor a synchronous control study, and the number of cases in the ICGVAL-MAP group was relatively small. We understand that the chronological improvement of the technique of the bronchoscopic procedure possibly contributed to the improvement in the outcomes of ICG-VAL-MAP. Moreover, the condition of the lung, such as anthracosis, emphysema, and interstitial pneumonia, might have influenced the visualization of the markings. Despite this, because there is no standard quantitative method for describing the optical characteristics of a lung surface, this aspect was not considered in the statistical analysis for this study. Further multicenter and randomized controlled studies that consider lung condition are warranted. Third, because the marking visibility grading, performed by the attending surgeons, was not blindly assessed, bias may present in the assessments. Although we established paper-based instructions on the evaluation of visibility before the study, further research with blinded assessment is warranted. Lastly, because ICG-VAL-MAP contained a CT contrast agent, which may not be applicable for patients with bronchial asthma and kidney failure, we need to be aware of the indications of ICGVAL-MAP. However, considering the intrapulmonary administration of the injection and relatively small amount of the CT contrast agent, no clinical complications might have occurred. However, further investigation on this aspect would be necessary in future studies.

TABLE 3. Complications in the 2 groups

\begin{tabular}{|c|c|c|c|}
\hline Complication & VAL-MAP & ICG-VAL-MAP & $P$ value \\
\hline Pneumothorax, pleural injury & 8 & 0 & .12 \\
\hline Fever & 7 & 2 & .72 \\
\hline Others & 1 (alveolar hemorrhage) & 1 (vagal reflex) & N/A \\
\hline
\end{tabular}

Values are presented as n. VAL-MAP, Virtual-assisted lung mapping; ICG-VAL-MAP, indocyanine green virtual-assisted ling mapping; N/A, not available. 
Characteristics of patients and markings

\begin{tabular}{|l|c|c|c|}
\hline & VAL-MAP & ICG-VAL-MAP & P value \\
\hline Cases & 147 & 63 & N/A \\
\hline Lesions & 228 & 77 & N/A \\
\hline Age, y.o. & $65(58-72)$ & $69(62-75)$ & 0.03 \\
\hline Sex, male/female & $35 / 28$ & $66 / 81$ & 0.15 \\
\hline Resected nodules & $227(99.6 \%)$ & $77(100 \%)$ & $>0.99$ \\
\hline Size of lesions (mm) median (IQR) & $8.0(4.5-12)$ & $8.0(6.0-13.9)$ & 0.82 \\
\hline Depth of lesions (mm) median (IQR) & $4.0(1-10)$ & $9.0(2.0-13.0)$ & 0.01 \\
\hline Number of markings & 567 & 142 & N/A \\
\hline Markings per nodule & $3(2-4)$ & $2(1-3)$ & $<0.0001$ \\
\hline
\end{tabular}

VIDEO 1. Brief description of the effectiveness of indocyanine green virtual-assisted ling mapping (ICG-VAL-MAP) in visualizing lung markings compared with that of virtual-assisted lung mapping (VAL-MAP). Video available at: https://www.jtcvs.org/article/S2666-2507(21)00498-3/ fulltext.

\section{CONCLUSIONS}

We revised the VAL-MAP as ICG-VAL-MAP with the use of a CT contrast agent with ICG. ICG-VAL-MAP showed clinical feasibility and improved visibility on CT and intraoperatively and may be useful for small lung nodule resection.

\section{Conflict of Interest Statement}

The authors reported no conflicts of interest.

The Journal policy requires editors and reviewers to disclose conflicts of interest and to decline handling or reviewing manuscripts for which they may have a conflict of interest. The editors and reviewers of this article have no conflicts of interest.

The authors thank Dr Kota Ono for providing statistical advice.

\section{References}

1. Saghir Z, Dirksen A, Ashraf H, Bach KS, Brodersen J, Clementsen PF, et al. CT screening for lung cancer brings forward early disease. The randomized Danish lung cancer screening trial: status after five annual screening rounds with lowdose CT. Thorax. 2012;67:296-301.
2. Chen-Yoshikawa TF, Fukui T, Nakamura S, Ito T, Kadomatsu Y, Tsubouchi H, et al. Current trends in thoracic surgery. Nagoya J Med Sci. 2020;82:161-74.

3. Sakiyama S, Kondo K, Matsuoka H, Yoshida M, Myoshi T, Yoshida S, et al. Fatal air embolism during computed tomography-guided pulmonary marking with a hook-type marker. J Thorac Cardiovasc Surg. 2003;126:1207-9.

4. Horan TA, Pinhreiro PM, Araujo LM, Santiago FF, Rodrigues MR. Massive gas embolism during pulmonary nodule hook wire localization. Ann Thorac Surg. 2002;73:1647-9.

5. Sato M, Omasa M, Chen F, Sato T, Sonobe M, Bando T, et al. Use of virtual assisted lung mapping (VAL-MAP), a bronchoscopic multisport dye-marking technique using virtual images, for precise navigation of thoracoscopic sublobar lung resection. J Thorac Cardiovasc Surg. 2014;147:1813-9.

6. Sato M, Yamada T, Menju T, Aoyama A, Sato T, Chen F, et al. Virtual-assisted lung mapping: outcome of 100 consecutive cases in a single institute. Eur J Cardiothorac Surg. 2015;47:e131-9.

7. Sato M, Kuwata T, Yamanashi K, Kitamura A, Misawa K, Imashimizu K, et al. Safety and reproducibility of virtual-assisted lung mapping: a multicentre study in Japan. Eur J Cardiothorac Surg. 2017;51:861-8.

8. Nakamura S, Goto M, Chen-Yoshikawa. Fluorescence-guided thoracic surgery. Available at: https://jovs.amegroups.com/article/view/63270. Accessed August 9, 2021.

9. Chen-Yoshikawa TF, Date H. Update on three-dimensional image reconstruction for preoperative simulation in thoracic surgery. J Thorac Dis. 2016;8(Suppl 3): S295-301.

10. Fox IJ, Wood EH. Indocyanine green: physical and physiologic properties. Proc Staff Meet Mayo Clin. 1960;35:732-44.

11. Mao Y, Chi C, Yang F, Zhou J, He K, Li H, et al. The identification of subcentimetre nodules by near-infrared fluorescence thoracoscopic systems in pulmonary resection surgeries. Eur J Cardiothorac Surg. 2017;52:1190-6.

12. Chen-Yoshikawa TF, Hatano E, Yoshizawa A, Date H. Clinical application of projection mapping technology for surgical resection of lung metastasis. Interact Cardiovasc Thorac Surg. 2017;25:1010-1.

13. Anayama T, Qiu J, Chan H, Nakajima T, Weersink R, Daly M, et al. Localization of pulmonary nodules using navigation bronchoscope and a near-infrared fluorescence thoracoscope. Ann Thorac Surg. 2015;99:224-30.

14. Mun M, Okumura S, Nakao M, Matsuura Y, Nakagawa K. Indocyanine green fluorescence-navigated thoracoscopic anatomical segmentectomy. J Vis Surg. 2017:3:80.

15. Misaki N, Chang SS, Gotoh M, Yamamoto H, Satoh K, Yokomise H. A novel method for determining adjacent lung segments with infrared thoracoscopy. $J$ Thorac Cardiovasc Surg. 2009;138:613-8.

16. Tokuno J, Chen-Yoshikawa TF, Nakao M, Matsuda T, Date H. Resection process map: a novel dynamic simulation system for pulmonary resection. J Thorac Cardiovasc Surg. 2020;159:1130-8.

17. Chen-Yoshikawa TF, Date H. Three-dimensional image in lung transplantation. Gen Thorac Cardiovasc Surg. 2018;66:19-26.

Key Words: indocyanine green, VAL-MAP, iVATS 